\title{
Harrison's interpretation of the cosmological redshift revisited
}

\author{
Valerio Faraoni \\ Physics Department, Bishop's University \\ 2600 College St., Sherbrooke, Québec, Canada J1M 1Z7 \\ Emailvfaraoni@ubishops.ca
}

\begin{abstract}
Harrison's argument against the interpretation of the cosmological redshift as a Doppler effect is revisited, exaggerated, and discussed. The context, purpose, and limitations of the interpretations of this phenomenon are clarified.
\end{abstract}




\section{Introduction}

There is still much debate on whether the cosmological redshift can be interpreted as a Doppler effect (in the sense of Special Relativity) due to the recessional motion of galaxies, as originally envisaged by Hubble, or whether this interpretation is incorrect [1, 2, 3, 4, 5]. Many current discussions are based on particular choices of coordinates or observers. We emphasize that coordinate-based statements are meaningless in a covariant theory such as General Relativity. Families of observers, on the contrary, are defined in a coordinate-independent way by their four-velocity fields (often, coordinates and observers are confused because geometrically-defined observers identify certain coordinate systems, those in which they are at rest, but the distinction should be kept in mind). Each observer will have its own interpretation of a certain physical phenomenon, which is perfectly legitimate for that observer. A different observer will have a different interpretation, which is legitimate as well. It is pointless to debate which interpretation is "the correct one": they are all correct. However, it may happen that strong physical reasons select a preferred family of observers which, in turn, selects a preferred interpretation.

The standard textbook derivation of the cosmological redshift for an observer and a light source at rest in comoving coordinates in a Friedmann-Lemaitre-Robertson-Walker (FLRW) universe does not require the discussion of the (four-)velocities of the source and observer. However, in principle, it does not prevent one from looking for an interpretation in more familiar terms, either. The Doppler effect is obtained in Special Relativity by a Lorentz transformation from a source to an observer in Minkowski space, in which it is legitimate to speak of the relative motion and relative velocity of a light source and an observer located at different spatial points. In a FLRW (or in any curved) space instead one cannot compare directly the four-velocities of a source and an observer located at different spatial points. It seems, therefore, that the interpretation of the cosmological redshift in terms of Doppler effect is ill-conceived from the start and that this redshift should be attributed entirely to the gravitational field of the universe. This interpretation is consistent: it is well known that even a static gravitational field causes frequency shifts in null rays propagating through it. The classic example of this phenomenon is the shift experienced by a photon propagating between a source and an observer both at rest at different radii in the Schwarzschild spacetime. However, even in this case, one could still give a formal representation in terms of an "effective Doppler effect" by thinking of the shift as being equivalent to the Doppler shift that would occur if the source (observer) were falling freely to the position of the observer (source), reaching it with non-zero velocity. Narlikar [6] has given a precise meaning to this idea; since in a curved spacetime one cannot compare four-velocities at different points, the best one can do 
is to parallel-transport the four-velocity of the source to the observer's location along the photon worldline, and then construct an effective Doppler formula at that point. This formula contains, as special cases, the Doppler effect of Special Relativity, the shift in Schwarzschild space, and the cosmological redshift. It is clear, however, that this procedure entails a fictitious, not a real "relative motion", especially when this method is applied to the case of Schwarzschild space. It seems more convenient to distinguish between "purely special-relativistic" Doppler shift caused by local motion in Minkowski space and "purely gravitational" shift of the kind experienced by a photon propagating between a source and an observer at rest in the Schwarzschild space. It is safe to say that, for the latter, the frequency shift is caused by the fact that the metric tensor $g_{\mu \nu}$ assumes different values at the different spacetime points where the source and the observer are located. Where does this place cosmological redshift then? It is easy to see why a naive interpretation of the resdshift in terms of Doppler effect tends to linger. If a source and an observer at a fixed comoving distance $d_{\text {comoving }}$ are locally at rest with respect to the cosmic substratum, they still experience what advocates of the Doppler interpretation could call a "relative motion" in the sense that their physical (proper) separation $d_{\text {physical }}=a(t) d_{\text {comoving }}$ (where $a(t)$ is the scale factor) changes with time. The derivative of this physical separation with respect to the comoving time is

$$
\dot{d}_{\text {physical }}=\dot{a} d_{\text {comoving }}=H d_{\text {physical }},
$$

i.e., Hubble's law, where $H \equiv \dot{a} / a$ is the Hubble parameter. It is tempting to interpret $\dot{d}_{\text {physical }}$ as a "velocity of recession" $v$, especially for nearby galaxies, producing a redshift factor $v / c$. However, this "relative velocity" is not obtained by comparing directly fourvelocities at distinct spacetime points: such an operation is not defined and giving a meaning to it requires Narlikar's [6] non-local procedure of parallel transport. Then, the redshift is unambiguously ascribed to the spacetime curvature and, while Narlikar writes down an effective Doppler formula, it is conceptually different from the relative motion between source and observer in a static Minkowski spacetime. The identification of the cosmological reshift factor $z$ with $v / c$, thinking of $v$ as a relative velocity in Minkowski space, would be arbitrary.

The purpose of this work is to discuss the limits of the interpretations of the cosmological redshift in order to gain a better understanding of this phenomenon. To this end we develop, exaggerate, and discuss an example proposed by Harrison [7], who considered a light source and an observer at rest in Minkowski space. The source emits a light ray and, while this propagates to the observer, space suddenly expands for a short time and then stops expanding before the observer receives the signal (with the spacetime becoming Minkowskian again). Harrison argues that there is cosmological redshift given 
by the usual formula

$$
z+1=\frac{a_{O}}{a_{S}}
$$

(where $a(t)$ is the scale factor of the FLRW metric and subscripts $\mathrm{O}$ and S denote observer and source, respectively) and that, because both source and observer are at rest during emission and detection, this redshift cannot be interpreted as a Doppler effect. This situation is a special case of the general situation contemplated in the derivation of the cosmological redshift. In the general situation, consider source and observer widely separated in space and time; then, it can be said without risking any interpretation that the redshift is a non-local effect due to the different values of the scale factor at emission and absorption. If, following Narlikar [6], one wants to parallel-transport the four-velocity of the source at the observer and construct an effective Doppler formula, one is still sampling the curvature of a wide region of spacetime and non-local effects. This rules out arguments advocating local motions based on the statement that any curved manifold can be locally approximated by its tangent space and, therefore, curvature effects can only be seen as Doppler effects because curvature is not sampled on such small regions. This would be akin to saying that the Riemann tensor $R_{\mu \nu \alpha \beta}$ vanishes at one point $p$ because space is locally flat while, in fact, $R_{\mu \nu \alpha \beta}(p) \neq 0$ but its effects are of higher order and are only felt when larger regions of spacetime are sampled. Spacetime is locally flat, but the cosmological redshift is a non-local effect due to the curvature of the large region of spacetime sampled by the ray between emission and detection. A Doppler interpretation based on local arguments is meaningless, while one based on Narlikar's parallel transport is technically correct, although its convenience is debatable.

We stress that eq. (1.2), together with its derivation, is not under discussion here; it is a standard and established prediction of the theory, and textbook material. What is under discussion is its interpretation. An interpretation is usually part of the baggage of a physical theory; the interpretation of the cosmological redshift depends on a chosen set of observers. A priori, therefore, different interpretations based on different observers are possible and they would seem to be equally valid. However, in a spatially homogeneous and isotropic universe there is a physically preferred set of observers-those that see the cosmic microwave background as homogeneous and isotropic-they select a preferred interpretation.

Harrison's example has been taken and adapted by Saulson to illustrate an analogous situation in a different context [8]. A gravitational wave impinges on a laser interferometer and causes a phase shift between the laser beams propagating in the two arms. Saulson's purpose is to answer a common objection and to demonstrate that, although the wavelength of the laser light and the interferometer's arm in which it propagates are stretched by the same amount, the gravitational wave is observable through the non- 
vanishing phase shift (different approaches to this problem can be found in Refs. [9, 10]. Saulson [8] correctly notes the analogy with Harrison's argument in cosmology and makes his case by considering a gravitational wave amplitude described by a step function.

On this line, it seems fit to revisit Harrison's argument and exaggerate it for clarity by assuming that the cosmic expansion takes place suddenly at a single instant of time. For simplicity, the spacetime metric is given by the spatially flat FLRW line element

$$
d s^{2}=-d t^{2}+a^{2}(t)\left(d x^{2}+d y^{2}+d z^{2}\right)
$$

in comoving coordinates $(t, x, y, z)$ and with scale factor $a(t)=1+\theta(t)$, where

$$
\theta(t)=\left\{\begin{array}{lll}
0 & \text { if } & t<0 \\
1 & \text { if } & t \geq 0
\end{array}\right.
$$

is the Heaviside step function. This describes a universe doubling its size abruptly at time $t=0$.

There are risks in adopting discontinuous metrics: the gravitational accelerations (Christoffel symbols) are impulsive, while the curvature tensor and its contractions the Ricci tensor and Ricci scalar may be ill-defined because, in general, they contain products of distributions. At best, the corresponding stress-energy tensors will also be distributional. In spite of these formal difficulties, discontinuous and even delta-like metrics have been considered long ago by Penrose [11. Such exact solutions of the Einstein equations can be obtained by cut-and-paste procedures in which different regions of spacetime (perhaps Minkowski spaces, as in our present example) are joined together with a suitable warp along an hypersurface [11]. Similar metrics have been studied extensively in the literature on exact plane gravitational waves [12] and it has also been discussed how to make sense of the product of distributions in General Relativity [13]. It is not too surprising, therefore, to see a discontinuous metric in Saulson's example which deals with gravitational waves [8]. Abstracting from the technical difficulties with the Riemann tensor, in the following we study the propagation of a null ray between a source and an observer which are at rest initially (when light is emitted) and after the expansion of the universe has stopped (when light is received by the observer)-we derive and discuss the corresponding redshift formula. The calculation does not require the consideration of the curvature. 


\section{Harrison's model revisited}

The metric tensor and its inverse are

$$
\left(g_{\mu \nu}\right)=\left(\begin{array}{cccc}
-1 & 0 & 0 & 0 \\
0 & 1+3 \theta & 0 & 0 \\
0 & 0 & 1+3 \theta & 0 \\
0 & 0 & 0 & 1+3 \theta
\end{array}\right), \quad\left(g^{\mu \nu}\right)=\left(\begin{array}{cccc}
-1 & 0 & 0 & 0 \\
0 & \frac{1}{1+3 \theta} & 0 & 0 \\
0 & 0 & \frac{1}{1+3 \theta} & 0 \\
0 & 0 & 0 & \frac{1}{1+3 \theta}
\end{array}\right),
$$

respectively. The only non-vanishing Christoffels symbols are

$$
\begin{aligned}
& \Gamma_{11}^{0}=\Gamma_{22}^{0}=\Gamma_{33}^{0}=\frac{3 \delta(t)}{2} \\
& \Gamma_{01}^{1}=\Gamma_{10}^{1}=\Gamma_{02}^{2}=\Gamma_{20}^{2}=\Gamma_{03}^{3}=\Gamma_{30}^{3}=\frac{3 \delta(t)}{2[1+3 \theta(t)]},
\end{aligned}
$$

where $\delta(t)$ denotes the Dirac delta.

Consider now a light source located at $x=L$ and an observer at $x=0$, both on the $x$-axis. The source emits a signal at time $t_{S}$, which is received at time $t_{O}$ by the observer, with $t_{S}<0<t_{O}$. The null ray propagates along the $x$-axis in the direction of decreasing $x$ and has four-tangent

$$
u^{\mu}=\frac{d x^{\mu}}{d \lambda}=\left(u^{0}, u^{1}, 0,0\right)
$$

where $\lambda$ is a parameter along the null geodesic. The normalization $g_{\mu \nu} u^{\mu} u^{\nu}=0$ yields

$$
u^{1}=-\frac{u^{0}}{\sqrt{1+3 \theta(t)}} .
$$

The negative sign is chosen because $u^{0}=d t / d \lambda>0$ corresponding to the parameter $\lambda$ increasing along the null geodesic, and the ray propagates in the direction of decreasing $x$. The zero component of the null geodesic equation

$$
\frac{d u^{\mu}}{d \lambda}+\Gamma_{\alpha \beta}^{\mu} u^{\alpha} u^{\beta}=0
$$


then yields

$$
\frac{d u^{0}}{d \lambda}=-\Gamma_{11}^{0}\left(u^{0}\right)^{2}=-\frac{3 \delta(t)\left(u^{0}\right)^{2}}{2[1+3 \theta(t)]} .
$$

By using the definition of $u^{0}$, it is seen that $d \lambda=d t / u^{0}$ and

$$
\frac{d u^{0}}{d t}=-\frac{3 u^{0} \delta(t)}{2[1+3 \theta(t)]}
$$

The integration of this equation along the null geodesic between the source $\mathrm{S}$ and the observer $\mathrm{O}$ yields 1

$$
\ln \left[\frac{u_{(O)}^{0}}{u_{(S)}^{0}}\right]=-\frac{3}{2} \int_{t_{S}}^{t_{O}} d t \frac{\delta(t)}{1+3 \theta(t)}=-\ln 2
$$

therefore,

$$
u_{(O)}^{0}=\frac{u_{(S)}^{0}}{2} .
$$

Light is emitted in flat spacetime and the four-tangent to the null ray at $\mathrm{S}$ is $u_{(S)}^{\mu}=$ $(1,-1,0,0)$, hence

$$
u_{(O)}^{0}=\frac{1}{2}
$$

The propagating photon has four-wavevector $k^{\mu}=\omega u^{\mu}=(\omega, \vec{k})$ in flat spacetime. For a photon emitted at the source with unit angular frequency, it is $k^{\mu}=u^{\mu}$ and the angular frequency measured by the observer is $k_{(O)}^{0}=u_{(O)}^{0}=1 / 2$. In fact, the angular frequency measured by any observer with four-velocity $v^{\mu}$ is $-k^{\mu} v_{\mu}$, and both source and observer have four-velocity $v^{\mu}=\delta^{0 \mu}$ in comoving coordinates. A photon emitted at $\mathrm{S}$ with angular frequency $\omega_{S}$ will have an angular frequency as measured by $\mathrm{O}$

$$
\omega_{O}=\frac{\omega_{S}}{2}
$$

i.e., light is redshifted by the sudden cosmic expansion at $t=0$. The redshift factor defined in terms of the wavelenghts $\lambda_{S, O}^{e}$. of the electromagnetic signal at the source and observer is

$$
z \equiv \frac{\lambda_{O}^{e m}-\lambda_{S}^{e m}}{\lambda_{S}^{e m}}=\frac{\lambda_{O}^{e m}}{\lambda_{S}^{e m}}-1=\frac{\omega_{S}}{\omega_{O}}-1=1 .
$$

\footnotetext{
${ }^{1}$ Note that one cannot simply use the property $\int_{t_{S}}^{t_{O}} f(t) \delta(t) d t=f(0)$ because $f(t)=[1+3 \theta(t)]^{-1}$ is not a test function continuous with all its derivatives; the integration is nevertheless straightforward.
} 
Note that $z+1=2$ and that the universe has doubled its size at $t=0$, so the usual formula for the cosmological redshift (1.2) is satisfied. As a check of this little calculation, one can integrate also the $x$-component of the null geodesic equation

$$
\frac{d u^{1}}{d \lambda}=-2 \Gamma_{01}^{1} u^{0} u^{1}=\frac{3 \delta(t)\left(u^{1}\right)^{2}}{\sqrt{1+3 \theta(t)}} .
$$

The integration between $\mathrm{S}$ and $\mathrm{O}$ as above yields

$$
u_{(O)}^{1}=\frac{u_{(S)}^{1}}{4}
$$

and, using $u_{(S)}^{\mu}=(1,-1,0,0)$, it is $u_{(O)}^{1}=-1 / 4$. This value of $u_{(O)}^{1}$ coincides with the one obtained from eq. (2.4) evaluated at $\mathrm{O}$, i.e., $u_{(O)}^{1}=-u_{(O)}^{0} / 2=-1 / 4$.

\section{Discussion}

The cosmological redshift factor (1.2) is a straightforward prediction of standard cosmology, but its interpretation seems to be still controversial, judging from the number of articles debating it. Our implementation of Harrison's example should shed some light here. According to Harrison [7] the cosmological redshift cannot be interpreted as a Doppler shift because both source and observer are at rest when the signal is emitted or received. This redshift is due to the fact that the scale factor (the only degree of freedom of the metric $g_{\mu \nu}$ ) assumes different values at the spacetime points $\mathrm{S}$ and O. Narlikar's procedure of parallel-transporting the source four-velocity along the null geodesic from $\mathrm{S}$ to $\mathrm{O}$ and constructing an effective non-local Doppler formula there [6] can be applied, but it is not particularly useful for understanding the physics involved: here we do have a source and an observer at rest in comoving coordinates. One could object that, after all, these source and observer are not truly at rest all the time: the comoving distance between $\mathrm{S}$ and $\mathrm{O}$ is $L$, while their physical (proper) separation

$$
d_{\text {physical }}(t)=a(t) L=\left\{\begin{array}{ccc}
L & \text { if } & t<0 \\
2 L & \text { if } \quad t \geq 0
\end{array}\right.
$$

doubles suddenly at $t=0$ with infinite "velocity" $\frac{d d_{p}}{d t}=L \delta(t)$. Here we assumed that source and observer return to rest after the universe has expanded, without worrying about whether this happens spontaneously or some entity forces them to do so. It is shown in the Appendix that they actually remain spontaneously at rest. 
In some sense, therefore, there has been some "relative motion" of S and O, but this is not a local motion in the sense of Special Relativity. The space in which $\mathrm{S}$ and $\mathrm{O}$ live has expanded, changing their proper separation, and this sudden "non-local motion" has redshifted the light.

It has been argued [1, 4] that the interpretation depends on the coordinate or gauge adopted; it is more correct to say that it depends on the set of observers adopted (the latter are defined in a coordinate-independent way by their four-velocities). It is certainly true that different observers, which define different coordinate systems (those in which they are at rest with their four-velocity components given by $v^{\mu}=\delta^{0 \mu}$ ), will have different interpretations of the same physics. Each one of this is a legitimate interpretation for that observer. While the redshift factor is an observer-invariant quantity, its interpretation is not. There are other examples in which, by choosing different observers, coordinate systems, or gauges, different interpretations of the same physics arise. In a laser-interferometric detector of gravitational waves one can choose a gauge in which the end mirrors move, and attribute the phase shift to the relative motions of the mirrors, which differ in the two arms; or one can choose the transverse-traceless (TT) gauge in which the mirrors are at rest in TT coordinates (but their proper separation changes) and attribute the phase shift to the different rates at which time elapses (a frequency shift effect again) and caused by the passing gravitational wave. Of course, the physics is gauge-invariant: the phase shift (a scalar, therefore gauge-invariant, quantity) is ultimately a tidal effect caused by the curvature tensor, which is also a gauge-invariant quantity. Another example is quantum mechanics, in which the Schrödinger, Heisenberg, and interaction pictures all provide different interpretations of the same physics, and the use of these different formalisms is a matter of convenience.

The cosmological redshift in a FLRW space is a geometric, non-local, coordinateinvariant effect. It seems that there is little point arguing in favour of one of its interpretations based on a set of observers versus another. However, in a FLRW space, there definitely is a set of physically preferred observers: they are the comoving observers who see the cosmic microwave background homogeneous and isotropic around them (apart from small temperature fluctuations). It is arguable whether an interpretation of the redshift formula (1.2) derived in such a clear way in textbooks is really necessary but, if one opts for choosing an interpretation, this should be tied to the physically preferred comoving observers. For the latter, the redshift is definitely gravitational and not due to an ill-defined recessional motion of galaxies.

There is still a difficulty to be dealt with. In Ref. [1] discussing the interpretation of the cosmological redshift, the Milne universe is considered as an example: this is a region of Minkowski space written in accelerated coordinates, which yields the line element of 
an open universe with linear scale factor $a(t)=t$,

$$
d s^{2}=-d t^{2}+t^{2}\left[d \chi^{2}+\sinh ^{2} \chi\left(d \theta^{2}+d \varphi^{2}\right)\right]
$$

Of course, a calculation of the Riemann tensor shows that it vanishes everywhere and that this is (a portion of) flat spacetime foliated using hyperbolic 3-surfaces 2 There is redshift, which is certainly not due to gravity (which is absent here); it is definitely a Doppler shift due to the fact that the observers at rest in $(t, \chi, \theta, \varphi)$ coordinates ("Milne observers") are moving away from each other when seen from observers at rest in Minkowski space. The latter are comoving observers, but perhaps one could say that also the Milne observers can be called "comoving"; this ambiguity in the term "comoving" arises because spacetime is empty and any observer can be said to be "comoving" with no matter. However, this is really a pathological example and its choice to discuss the interpretation of the cosmological redshift is an unhappy one. A set of geometrically preferred observers consists of those with four-velocity $v^{\nu}$ parallel to the timelike Killing field of this metric, which is defined in an invariant way.

If the energy density $\rho$ of a FLRW universe is non-zero, there will be precisely one set of physically preferred observers that see zero spatial gradients of $\rho$ (comoving observers) and the ambiguity in the interpretation of the cosmological redshift (gravitational versus Doppler) disappears.

\section{Acknowledgments}

This work is supported by the Natural Sciences and Engineering Research Council of Canada.

\footnotetext{
${ }^{2}$ The spatial curvature is negative, but the spacetime curvature is zero.
} 


\section{Appendix}

Here we address the question of whether two objects initially at rest remain at rest or are set in relative motion after the expansion of the universe has stopped. Consider an observer with timelike four-velocity $v^{\mu}$ satisfying the initial condition $v_{\text {initial }}^{\mu}=(1,0,0,0)$ at $t<0$ and the normalization $g_{\mu \nu} v^{\mu} v^{\nu}=-1$ (this could be the source $\mathrm{S}$ or the observer $\mathrm{O}$ ). The geodesic equation yields

$$
\begin{aligned}
& \frac{d v^{0}}{d \lambda}=-\left[\Gamma_{11}^{0}\left(v^{1}\right)^{2}+\Gamma_{22}^{0}\left(v^{2}\right)^{2}+\Gamma_{33}^{0}\left(v^{3}\right)^{2}\right]=-\frac{3(\vec{v})^{2} \delta(t)}{2}, \\
& \frac{d v^{i}}{d \lambda}=-\frac{3 \delta(t) v^{0} v^{i}}{1+3 \theta(t)}
\end{aligned}
$$

where $(\vec{v})^{2}=\left(v^{1}\right)^{2}+\left(v^{2}\right)^{2}+\left(v^{3}\right)^{2}$. The normalization of $v^{\mu}$ yields

$$
(\vec{v})^{2}=\frac{\left(v^{0}\right)^{2}-1}{1+3 \theta(t)}
$$

Using eq. (3.5) and $d \lambda=d t / v^{0}$ along the timelike geodesic with tangent $v^{\mu}$, and integrating between times $t_{\text {initial }}$ and $t_{\text {final }}$, one obtains

$$
v_{\text {final }}^{i}=\frac{v_{\text {initial }}^{i}}{1+3 \theta(t)}= \begin{cases}v_{\text {initial }}^{i} & \text { if } t<0 \\ \frac{v_{\text {initial }}^{i}}{4} & \text { if } t \geq 0\end{cases}
$$

The initial condition $v_{\text {initial }}^{i}=0$ then guarantees that $v^{i}=0$ at all times. Indeed, due to spatial isotropy, an object initially at rest cannot pick up a spatial velocity as a consequence of the sudden cosmic expansion because this would select a preferred direction in 3-space. 


\section{References}

[1] R.J. Cook and M.S. Burns 2009, Am. J. Phys. 59, 59.

[2] M.J. Chodorowski, arXiv:astro-ph/0601171.

[3] M.J. Chodorowski 2007, Mon. Not. R. Astr. Soc. 378, 239.

[4] E.F. Bunn and D.W. Hogg, arXiv:0808.1081.

[5] M.J. Francis, L.A. Barnes, J.B. James, and G.F. Lewis 2007, Publ. Astron. Soc. Austral. 24, 95.

[6] J.V. Narlikar 1994, Am. J. Phys. 62, 903.

[7] E.R. Harrison 2000, Cosmology, The Science of The Universe, 2nd edition (CUP, Cambridge), pp. 317-319.

[8] P.R. Saulson 1997, Am. J. Phys. 65, 501.

[9] D. Garfinkle 2006 Am. J. Phys. 174, 196.

[10] V. Faraoni 2007, Gen. Rel. Gravit. 39, 677.

[11] R. Penrose 1972, in General Relativity, Papers in Honour of J.L. Synge, L. O'Raifeartaigh editor (Clarendon Press, Oxford), pp. 101-115.

[12] V. Ferrari, P. Pendenza, and G. Veneziano 1988, Gen. Rel. Gravit. 20, 1185; R. Steinbauer 1998, J. Math. Phys. 39, 2201; J. Podolsky, arXiv:gr-qc/0201029; M. Ortaggio and J. Podolski 2002, Class. Quantum Grav. 19, 5221; J. Podolsky and R. Steinbauer 2003, Phys. Rev. D 67, 064013.

[13] R. Steinbauer and J.A. Vickers 2006, Class. Quantum Grav. 23, R91-R114. 